\title{
QUASISYMMETRIC FUNCTIONS WITH DILATATION ONE
}

\author{
MATTI LEHTINEN
}

In a recent paper [3] Strebel introduced the dilatation of a homeomorphism of a Jordan curve onto another as follows: Let $G_{j}, j=1,2$, be Jordan domains and $\varphi: \partial G_{1} \rightarrow \partial G_{2}$ a sense-preserving homeomorphism. Consider all ring domains $A_{j} \subset G_{j}$ such that one boundary component of $A_{j}$ is $\partial G_{j}$, and quasiconformal mappings $f: A_{1} \rightarrow A_{2}$ such that $f \mid \partial G_{1}=\varphi$. The infimum of the maximal dilatations of all such mappings is called the interior dilatation of $\varphi$. The exterior dilatation is defined similarly using ring domains in the complements of $G_{1}$ and $G_{2}$. If $\partial G_{1}$ and $\partial G_{2}$ are analytic, the interior and exterior dilatations of $\varphi$ coincide. We then call their common value the dilatation of $\varphi$ and denote it by $L(\varphi)$.

Assume $L(\varphi)<\infty$ and denote by $Q_{\varphi}$ the class of all quasiconformal mappings $g: G_{1} \rightarrow G_{2}$ such that $g \mid \partial G_{1}=\varphi$. Making use of a well-known extension theorem [2, p. 96] one easily concludes that $Q_{\varphi} \neq \emptyset$. The class $Q_{\varphi}$ contains one or more extremals, i.e. mappings with smallest possible maximal dilatation in $Q_{\varphi}$. Denote this dilatation by $K(\varphi)$. It was shown by Strebel that if $L(\varphi)<K(\varphi)$, then $Q_{\varphi}$ contains only one extremal, which is a Teichmüller mapping. In particular, then, if $L(\varphi)=1$, the extremal is always unique, and either conformal or a Teichmüller mapping.

Strebel [3, p. 469] obtained a necessary and sufficient condition for $\varphi$ to have dilatation one in the case $G_{1}=G_{2}=$ the unit disc. There is, however, some interest in carrying Strebel's characterization over to the case of the upper half-plane, the boundary mappings then being the familiar quasisymmetric functions.

Now let $\varphi: \boldsymbol{R} \rightarrow \boldsymbol{R}$ be an increasing homeomorphism. It gives rise to a function $q_{\varphi}: H \rightarrow \boldsymbol{R}_{+}$, defined by

$$
q_{\varphi}(x+i y)=\frac{\varphi(x+y)-\varphi(x)}{\varphi(x)-\varphi(x-y)} .
$$

Thus $\varphi$ is $k$-quasisymmetric if $q_{\varphi}$ is bounded above by $k$ and below by $1 / k$. Whether $\varphi$ has dilatation one depends on the behavior of $q$ near the real axis:

Theorem 1. An increasing homeomorphism $\varphi: \boldsymbol{R} \rightarrow \boldsymbol{R}$ has dilatation one if and only if $q_{\varphi}(z)$ tends to one as $z$ tends to the real axis in the spherical metric. 
Proof. To prove the sufficiency part, we utilize the construction of Beurling and Ahlfors [1]. Given an arbitrary $\eta>1$, there exist positive numbers $m, M$ such that

$$
\eta^{-1}<q_{\varphi}(z)<\eta
$$

for all $z$ in the subset $E$ of $H$ whose elements satisfy $|\operatorname{Re} z|>M, \operatorname{Im} z<m$, or $\operatorname{Im} z>M$. For all $z=x+i y \in H$ set

$$
\alpha_{j}(z)=\int_{0}^{1} \varphi\left(x+(-1)^{j} y t\right) d t, \quad j=0,1 .
$$

Then $\alpha_{j}$ is differentiable, and its partial derivatives are

Now set

$$
\begin{aligned}
& \left(\alpha_{j}\right)_{x}(z)=(-1)^{j}\left(\varphi\left(x+(-1)^{j} y\right)-\varphi(x)\right), \\
& \left(\alpha_{j}\right)_{y}(z)=\int_{0}^{1}(-1)^{j} t d \varphi\left(x+(-1)^{j} y t\right) .
\end{aligned}
$$

$$
f(z)=(1 / 2)\left(\alpha_{0}(z)+\alpha_{1}(z)+i\left(\alpha_{0}(z)-\alpha_{1}(z)\right) .\right.
$$

It follows from the hypothesis and the continuity of $q_{\varphi}$ that $\varphi$ is $k$-quasisymmetric for some $k$. By [1], $f$ is a quasiconformal homeomorphism of $H$, agreeing with $\varphi$ on $\boldsymbol{R}$. The dilatation quotient $D$ of $f$ at $z$ satisfies

where

$$
D+D^{-1}=\frac{5\left(1+\xi_{0}^{2}\right) \zeta+5\left(1+\xi_{1}^{2}\right) / \zeta+6\left(\xi_{0} \xi_{1}-1\right)}{4\left(\xi_{0}+\xi_{1}\right)},
$$

$$
\zeta=\left(\alpha_{1}\right)_{x}(z) /\left(\alpha_{0}\right)_{x}(z), \quad \xi_{j}=(-1)^{j}\left(\alpha_{j}\right)_{y}(z) /\left(\alpha_{j}\right)_{x}(z),
$$

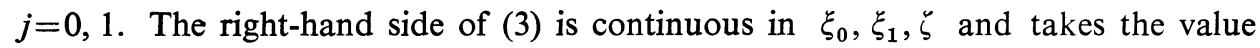
2 for $\xi_{0}=\xi_{1}=1 / 2, \zeta=1$. In order to have $D$ arbitrarily close to one it thus suffices to have $\xi_{0}$ and $\xi_{1}$ sufficiently close to $1 / 2$ and $\zeta$ sufficiently close to 1 . Now $\zeta=1 / q_{\varphi}(z)$ so that $1 / \eta \leqq \zeta \leqq \eta$ holds in $E$. We next estimate $\xi_{0}$. By a lemma of Beurling and Ahlfors [1, p. 137]

$$
\frac{1}{1+\eta} \leqq \xi_{0}(x+i y) \leqq \frac{\eta}{1+\eta},
$$

provided $\varphi$ is $\eta$-quasisymmetric in the interval $(x, x+y)$. This is certainly true if the triangle with vertices $x, x+y, x+y / 2+i y / 2$ is contained in $E$. Suppose then that this is not the case. First assume $x \geqq-M, y \geqq 2 M$. By (1) and (2),

$$
\xi_{0}(x+i y)=\frac{\int_{0}^{1}(\varphi(x+y)-\varphi(x+t y)) d t}{\varphi(x+y)-\varphi(x)},
$$


and since $x+i y \in E$,

or

$$
\eta^{-2} \xi_{0}(x+i y) \leqq \frac{\int_{0}^{1}(\varphi(x+y+y t)-\varphi(x+y)) d t}{\varphi(x+2 y)-\varphi(x+y)} \leqq \eta^{2} \xi_{0}(x+i y)
$$

$$
\eta^{-2} \xi_{0}(x+i y) \leqq 1-\xi_{0}(x+y+i y) \leqq \eta^{2} \xi_{0}(x+i y) .
$$

Our assumption implies that $\xi_{0}(x+y+i y)$ satisfies (4), and hence

$$
\frac{1}{\eta^{2}(1+\eta)} \leqq \xi_{0}(x+i y) \leqq \frac{\eta^{3}}{1+\eta} .
$$

If $x \leqq-M, y \geqq 2 M$, we may write

$$
\xi_{0}(x+i y)=1-\frac{\int_{0}^{1}(\varphi(x+y t)-\varphi(x)) d t}{\varphi(x+y)-\varphi(x)},
$$

and a similar argument yields

$$
\frac{1+\eta-\eta^{3}}{1+\eta} \leqq \xi_{0}(x+i y) \leqq \frac{\eta^{3}+\eta^{2}-1}{\eta^{2}(\eta+1)} .
$$

Completely analogous estimates hold for $\xi_{1}$. It follows that given $K>1$, we can always find $m$ and $M$ such that $f$ is $K$-quasiconformal outside the trapezoid with vertices $\pm M+i m, \pm(3 M-m)+2 M i$. By definition, then, $L(\varphi)=1$.

On the other hand, assume $L(\varphi)=1$. Let $\eta>1$ be arbitrary. By a lemma of Strebel [3, p. 469] there exists a $\delta>0$ such that

$$
\eta^{-1} \leqq q_{\varphi}(x+i y) \leqq \eta
$$

as soon as $0<y<\delta$. We thus have to estimate $q_{\varphi}(z)$ only for $\operatorname{Im} z \geqq \delta$ and $|z|$ large. There is no loss of generality in supposing $x \geqq 0$. By assumption, there exist positive numbers $m, M$ such that $\varphi$ can be extended to an $\eta$-quasiconformal mapping $f$ of the set $E$ considered in the first part of the proof. Further let $E_{j}$, $j=0,1$, be the simply connected domain obtained from $E$ by removing the closed rectangle with vertices $0,(-1)^{j} M,(-1)^{j} M+i m, i m$. We consider three cases: (i) $0 \leqq x \leqq M$, (ii) $x>M$ and $x>y$, (iii) $x>M$ and $x \leqq y$.

In case (i), take $y \geqq 2 M$ and consider the quadrilateral $E_{1}(x-y, x, x+y, \infty)$. The mapping $\zeta \mapsto(\zeta-x) / y$ transforms it into a quadrilateral $E_{z}(-1,0,1, \infty)$ without changing the conformal module. It is clear that as $y \rightarrow \infty$ the distance of any point lying on the side of $E_{z}$ with endpoints $-1,0$ from the line segment joining the same points approaches zero. By the continuity of the module (see e.g. 
[2, p. 26]), $\bmod E_{z}$ tends to $\bmod H(-1,0,1, \infty)=1$. In case (ii) the spherical distance of the side of $E_{z}$ with endpoints $-\infty,-1$ from the ray $(-\infty,-1)$ tends to zero as $|z| \rightarrow \infty$. (Observe that we assume $y \geqq \delta$.) Consequently $\bmod E_{1}(x-y, x, x+y, \infty)=\bmod E_{z}(-1,0,1, \infty) \rightarrow 1$. In case (iii), a similar argument yields $\bmod E_{0}(x-y, x, x+y, \infty) \rightarrow 1$ as $|z| \rightarrow \infty$. The same argument also shows that $\bmod f\left(E_{j}\right)(\varphi(x-y), \varphi(x), \varphi(x+y), \infty)$ (where $j=1$ in cases (i) and (ii), and $j=0$ in case (iii)) tends to $\bmod H(\varphi(x-y), \varphi(x), \varphi(x+y), \infty)$ as $|z| \rightarrow \infty$. Thus for $|z|$ large enough

$$
\begin{aligned}
\eta^{-3} & \leqq \eta^{-2} \bmod E_{j} \leqq \eta^{-1} \bmod f\left(E_{j}\right) \\
& \leqq \bmod H(\varphi(x-y), \varphi(x), \varphi(x+y), \infty) \\
& \leqq \eta \bmod f\left(E_{j}\right) \leqq \eta^{2} \bmod E_{j} \leqq \eta^{3} .
\end{aligned}
$$

But this means that for $|z|$ large enough

$$
\lambda\left(\eta^{3}\right)^{-1} \leqq q_{\varphi}(z) \leqq \lambda\left(\eta^{3}\right),
$$

where $\lambda$ is the distortion function defined in [2, p. 81]. As $\lim _{t \rightarrow 1} \lambda(t)=1$, and because of (5), the theorem is proved.

It follows at once from the extension theorem of Beurling and Ahlfors or from the above proof that $k$-quasisymmetric mappings with a small $k$ necessarily have a small dilatation. It is, however, easy to construct examples showing that the converse is not in general true:

Theorem 2. There exist homeomorphisms $\varphi: \boldsymbol{R} \rightarrow \boldsymbol{R}$ with $L(\varphi)=1$ and $\max _{z \in H} q_{\varphi}(z)$ arbitrarily large.

Proof. Given $M>0$, set

$$
\begin{array}{ll}
\psi(x)=8 M\left(x^{3}-x^{4}\right), & 0 \leqq x \leqq 1 / 2, \\
\psi(x)=M-\psi(1-x), & 1 / 2<x \leqq 1, \\
\psi(x)=\psi(1-(x-1) /(3 M)), & 1<x \leqq 3 M+1,
\end{array}
$$

and $\psi(x)=0$ otherwise. Then $\psi$ has a continuous second derivative, and $\psi^{\prime}(x) \geqq$ $\geqq \psi^{\prime}(1+3 M / 2)=-2 / 3$. Set $\varphi(x)=x+\psi(x)$. Then $\varphi: \boldsymbol{R} \rightarrow \boldsymbol{R}$ is increasing, $\varphi(1)=1+M, q_{\varphi}(i)=M+1$. Let $K$ be an upper bound for $\psi^{\prime \prime}$. Then $q_{\varphi}(x+i y)=$ $=\varphi^{\prime}\left(\xi_{1}\right) / \varphi^{\prime}\left(\xi_{2}\right)$ is bounded above by $\left(\varphi^{\prime}(x)+K y\right) /\left(\varphi^{\prime}(x)-K y\right)$ and below by $\left(\varphi^{\prime}(x)-K y\right) /\left(\varphi^{\prime}(x)+K y\right)$, whence $\lim _{y \rightarrow 0} q_{\varphi}(x+i y)=1$, uniformly in $x$. It is clear that $q_{\varphi}(z)$ tends to one as $|z| \rightarrow \infty$. By Theorem $1, L(\varphi)=1$. 


\title{
References
}

[1] Beurling, A., and L. V. Ahlfors: The boundary correspondence under quasiconformal mappings. - Acta Math. 96, 1956, 125-142.

[2] Lehto, O., and K. I. Virtanen: Quasiconformal mappings in the plane. - Springer-Verlag, Berlin - Heidelberg - New York, 1973.

[3] Strebel, K.: On the existence of extremal Teichmueller mappings. - J. Analyse Math. 30, 1976, 464-480.

\author{
University of Helsinki \\ Department of Mathematics \\ SF-00100 Helsinki 10 \\ Finland
}

Received 9 February 1977 